\title{
Le nom propre dans les manuscrits saussuriens
}

\author{
Stefania Montes HENRIQUES \\ Université Fédérale d'Uberlandia (ILEEL/PPGEL) \\ temontess@gmail.com
}

\section{Introduction}

Le Cours de Linguistique Générale ${ }^{1}$, édité à partir de certains manuscrits de Ferdinand de Saussure ainsi qu'un certain nombre de notes de ses étudiants établissant la langue comme objet d'étude a été considéré comme la base de la linguistique. Mais, qu'est-ce que la langue ? La langue, pour Saussure, est un système qui se fonde sur le rapport entre ses constituants, ceci implique une valorisation de l'étude de l'intérieur de ce système et non pas de ses relations extérieures.

On peut affirmer que définir la langue comme système autonome est contrarier les divers courants linguistiques et philosophiques qui ont établi un rapport entre les signes et leurs référents. Parmi ces courants, il existe la théorie naïve de la référence qui défend que les mots d'une langue se réfèrent directement aux objets qu'ils désignent, sans interférence sémantique ; c'est-à-dire que le lien entre les mots et les objets n'est pas définit par le sens et, de ce point de vue, la langue est considérée une nomenclature.

La critique saussurienne de cette conception est formulée dans le CLG : «Pour certaines personnes la langue, ramenée à son principe essentiel, est une nomenclature, c'est-à-dire une liste de termes correspondant à un certain nombre de choses » (SAUSSURE, p. 79, 1973) page 97. De cette façon, Saussure nie que la langue ne soit que la liste des objets existants dans le monde. Cependant, nier la langue comme nomenclature n'est pas nier qu'une certaine catégorie de signes ait une correspondance avec des objets. Sur ce point, nous sommes d'accord avec Chiesa (2008) :

\begin{abstract}
Mais le rejet de la conception du langage comme une nomenclature n'implique pas le rejet de la nomenclature en tant que cas particulier du langage et par conséquent d'une série de mots, à savoir les noms, qui comportent une référence à des objets dont les locuteurs sont conscients. Il s'agit dès lors de réexaminer la question de la nomenclature dans les écrits de Saussure. (CHIESA, p. 11, 2008)
\end{abstract}

Ce besoin de réexaminer la question de la nomenclature dans les manuscrits saussuriens est dû au fait que, bien que le nom propre ne soit cité qu'une fois dans le Cours de Linguistique Générale2, plusieurs indices montrent que Saussure s'est consacré à l'étude des toponymes et de l'onymique, au début du XXe siècle, en particulier, selon Testenoire (2008) durant la période comprise entre 1900 et 1904.

(...) la question du nom propre a été, depuis le début des années 1900, au cœur des travaux scientifiques de Ferdinand de Saussure. De 1900 à 1904, en effet, toutes ses communications scientifiques sont consacrées à l'étude des toponymes de la région genevoise. (TESTENOIRE, p. 1006, 2008). 
Les travaux scientifiques auxquels Testenoire (2008) se réfère sont, principalement, deux allocutions prononcées à la Société d'Histoire et d'Archéologie de Genève et intitulées : "Le nom de la ville d'Oron à l'époque romaine" (1901) et "Origine de quelques noms de lieux de la région genevoise"(1903)3. Il convient d'affirmer à propos de cette production scientifique saussurienne qui inclut essentiellement, la question des toponymes, qu'elle part d'une perspective diachronique et, met en évidence une étude de la référence de la part du genevois. Outre ces études, il existe aussi des éléments dans les recherches saussuriennes qui se réfèrent à la pratique anagrammatique et à des légendes germaniques, qui sont centrées sur la question des noms propres.

Malgré le bien-fondé de ces travaux par rapport à notre thème, nous avons choisi de traiter, dans cet article, les considérations faites par Saussure sur les noms propres et les toponymes dans le manuscrit « Notes Item. Sôme et sème » qui se trouve dans l'archive Ms. Fr. 3951, à la Bibliothèque publique de Genève $^{4}$. Ce manuscrit n'est pas daté précisément, mais selon Testenoire (2008), et considérant qu'il semble être une réaction à la lecture de l' «Essai de Sémantique » de Michel Bréal, nous supposons qu'il était inséré dans les productions saussuriennes du début du XXe siècle. Puis nous aborderons certains extraits $^{5}$ de manuscrits qui relatent les études faites par Saussure au sujet des légendes germaniques, archivées avec les références Ms. Fr. 3958 et Ms. Fr. 3959, qui contiennent des observations plus approfondies sur les noms propres

Avant de développer nos observations, il est nécessaire d'éclaircir quelques aspects de notre analyse et également, quelques critères qui y seront utilisés. D'abord, bien que le manuscrit examiné ait été édité et publié par S. Bouquet et R. Engler, dans l' "Écrits de Linguistique Générale », nous avons choisi d'analyser le manuscrit et non son édition. Ce choix se justifie dans la mesure où nous avons l'intention de déceler le processus de l'élaboration de la production saussurienne sur les noms propres et géographiques, tout en mettant l'accent sur les incises et les ratures effectuées par le genevois. De cette façon, nous avons l'intention de mettre en lumière quelques considérations saussuriennes pertinentes sur la question du nom propre et, en conséquence, sur la question de la référence.

\section{Les noms dans le CLG : des mots isolés}

Comme cela a été dit précédemment, il y a une négation explicite de la conception de la langue comme nomenclature dans le CLG. Cette critique sera, effectivement, reprise plusieurs fois au cours du développement de l'argumentation saussurienne. On peut affirmer par exemple que, quand Saussure se penche sur la nature du signe linguistique et le détermine comme l'union arbitraire de deux entités psychiques, il établit déjà que le référent ne fait pas partie du fonctionnement linguistique.

En outre, il nous semble que l'arbitraire est le principe directionnel ${ }^{6}$ de cette séparation $^{7}$ de la référence par rapport au signe linguistique. Ceci parce que quand il effectue le déplacement du principe de l'arbitraire à l'intérieur du signe, c'est-à-dire, entre le signifiant et le signifié, Saussure sépare le référent de la constitution du signe linguistique. Cette position est contraire, par exemple, à celle défendue dans l'Antiquité Classique, durant laquelle on affirmait que le lien entre les noms et les choses avait le statut d'arbitraire. Selon Gadet (1990), le principe de l'arbitraire aurait deux faces : l'une linguistique (entre signifiant et signifié) et l'autre philosophique (entre signe et objet) (cf. GADET, 1990 apud SILVA, p. $41)^{8}$.

À propos de ce principe, Saussure (1973) affirme que :

Le principe de l'arbitraire du signe n'est contesté par personne ; mais il est souvent plus aisé de découvrir une vérité que de lui assigner la place qui lui revient. Le principe énoncé plus haut domine toute la linguistique de la langue ; ses conséquences sont innombrables. (SAUSSURE, 1973, p.82) 
On peut considérer que quand il affirme « il est souvent plus aisé de découvrir une vérité que de lui assigner la place qui lui revient », Saussure critique, une fois encore, la conception de la langue comme nomenclature - essentiellement aristotélique - car si la langue était une liste de noms qui font référence à des objets déterminés, ce lien serait établi de façon arbitraire. Selon les notes d'Émile Constantin, qui concernent le troisième cours (1910-1911) donné par Saussure à Genève, penser la langue comme nomenclature serait infantile: "C'est une méthode enfantine. Si nous l'adoptons pour un moment, nous verrons facilement en quoi consiste le signe linguistique et en quoi il ne consiste pas." (E. CONSTANTIN, apud ENGLER, p. 148, 1989).

Mais, et le nom propre ? De quelle façon apparaît-il dans la théorisation saussurienne du CLG dans la mesure où cette oeuvre contient une critique intense à la conception de la langue comme nomenclature ? C'est dans la partie consacrée à la linguistique diachronique, dans le chapitre référent à l'analogie, qu'apparaît le seul commentaire référent au nom propre dans le CLG. Ce commentaire n'explique pas le problème du rapport entre les noms et les objets qui d'ailleurs, n'est pas cité :

Les seules formes sur lesquelles l'analogie n'ait aucune prise sont naturellement les mots isolés, tels que les noms propres spécialement les noms de lieu (cf. Paris, Genève, Agen etc.), qui ne permettent aucune analyse et par conséquent aucune interpretation de leurs eléments.(SAUSSURE, 1967, p. 237)

Donc, il n'y a aucune considération plus profonde sur la nature ou la fonction des noms propres partant de la considération que la langue est un système qui possède un ordre propre. Ce qui est intéressant dans cette citation, c'est que les noms propres sont considérés comme des mots isolés ; affirmation qui semble indiquer que cette catégorie linguistique constitue une exception à la théorie des signes, puisqu'elle/ n'établit pas de rapports « horizontaux » avec les autres signes du système.

Ainsi, dans le CLG, les considérations de Saussure sur le nom propre se restreignent à la critique de la langue comme nomenclature et à l'impossibilité de la soumission de cette catégorie linguistique face au phénomène de l'analogie, ce qui n'arrive pas avec les manuscrits saussuriens. Dans cet article, précisément, on analysera des extraits du manuscrit «Notes Item. Sôme et séme », dans lesquels la question de la référence et la spécificité des noms propres et des noms géographiques est mieux développée par Saussure.

\section{Le noms et l'objet : un rapport vertical}

Selon Chiesa (2008), Saussure indiquerait, implicitement, deux types de rapports établis par le signe linguistique. L'un serait de nature horizontale et se présenterait dans un système linguistique, établi entre un signe et les autres signes qui l'entoure. Nous pouvons affirmer que, ce rapport est le même que celui stipulé dans la théorie de la valeur. L'autre type de rapport serait de nature verticale et comprendrait la relation entre les noms propres/toponymes et les objets auxquels ces noms font référence. (cf. CHIESA, p. $12,2008)$

Considérant que c'est le rapport vertical établi entre un signe et un objet qui nous intéresse dans cet article, nous passerons à l'analyse des extraits sélectionnés du manuscrit « Notes Item », qui décrit la conception des noms propres et de leurs rapports avec le référent, dans la perspective saussurienne. 
Congrès Mondial de Linguistique Française - CMLF 2012

SHS Web of Conferences 1 (2012)

SHS Web of Conferences

DOI $10.1051 /$ shsconf $/ 20120100327$

(C) aux auteurs, publié par EDP Sciences, 2012

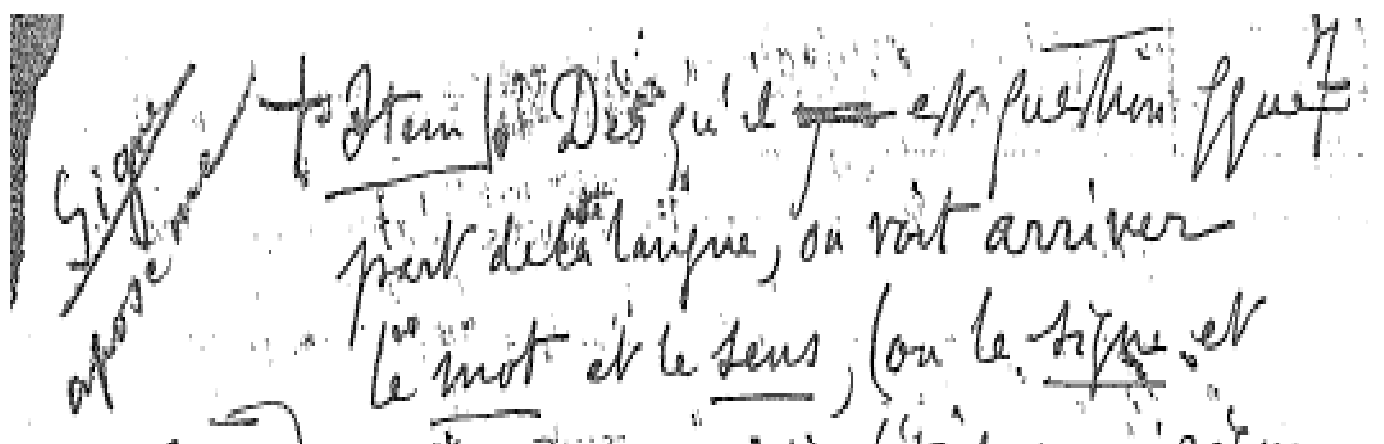

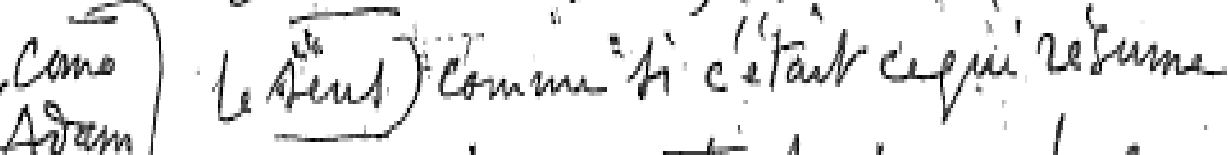

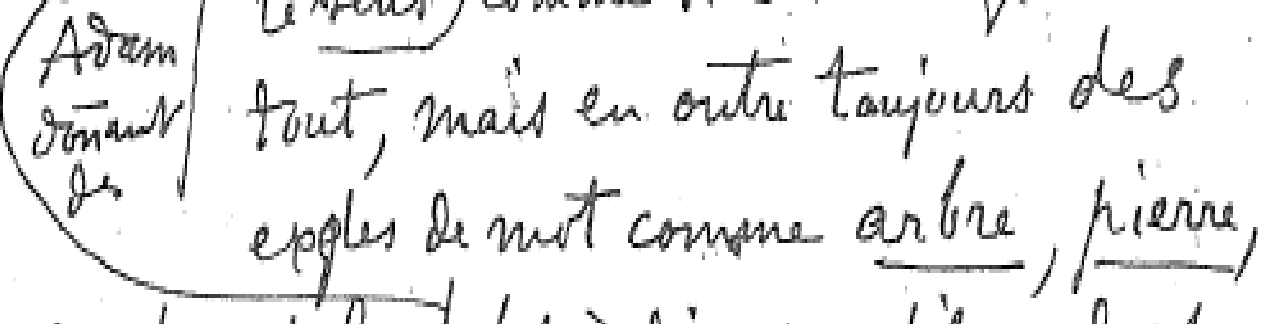

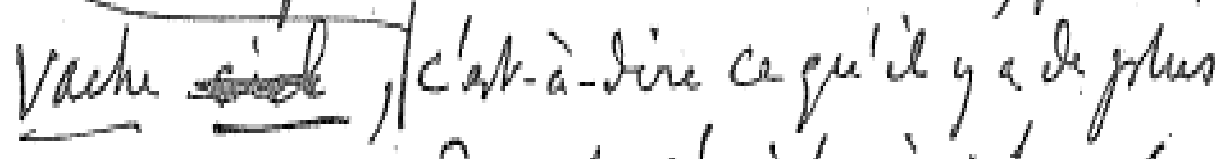
Cromier daw la semidogie: be cas on elle en (par la hasand des bijts

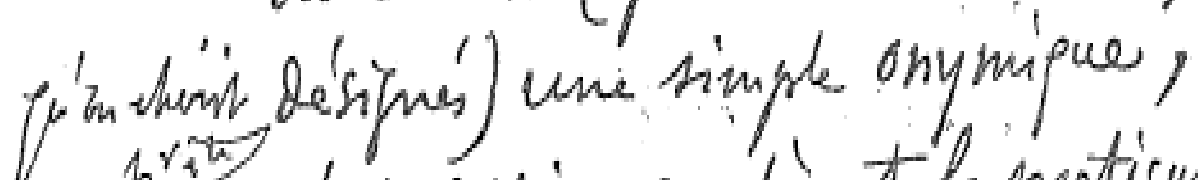

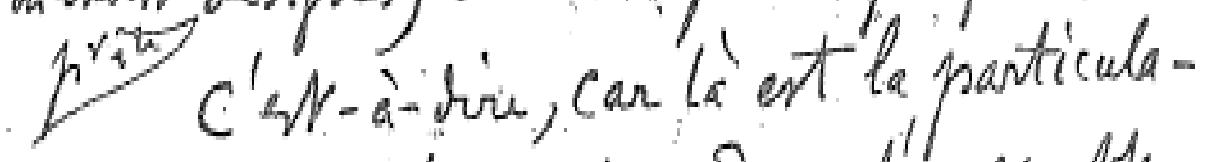

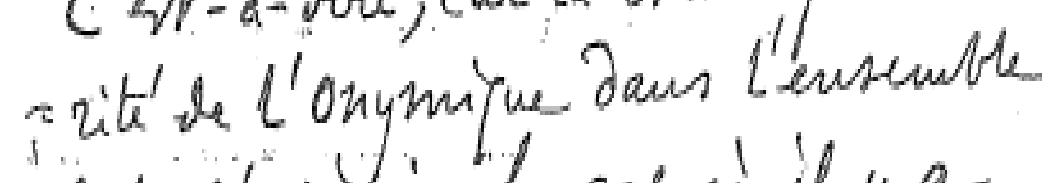
de semintipe, le cas ou lly a un troisieme elaments incontertade Dama L'association. prycholugiun du same, la conscience su'us'applíus à un être exteneur

any defimi en lni-mêmo prour échapper à la taigen'.

760

* s rale du hipus.

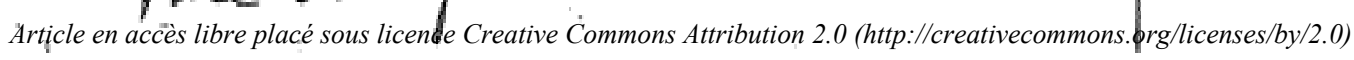


Figure 1. "Notes Item", p. 7

Cet extrait est extrêmement significatif dans la mesure où, quand il critique la langue comme nomenclature, Saussure admet « ce qu'il y a de plus grossier dans la sémiologie », qui est le phénomène de l'onymique. Un autre aspect intéressant à être considéré est le fait que les noms propres/toponymes ne sont pas cités alors que les noms communs comme « vache », « arbre », «pierre » et « ciel » (qui est raturé) le sont. La question que l'on se pose est: Pour quel motif Saussure inclut ces mots dans le phénomène de l'onymique si, dans le CLG, des mots de même type sont utilisés comme des exemples du principe de l'arbitraire et de la théorie de la valeur? L'exemple classique, cité postérieurement par Benveniste (2005), dans lequel Saussure se sert de la différence entre les termes boeuf et ochs pour confirmer, par l'intermédiaire des différentes langues, le principe de l'arbitraire fortifie notre sentiment d'inconfort dans la mesure où le signe « boeuf » est de même nature que le signe « vache » ou « arbre ».

De plus, on lit dans la première incise sur le côté gauche de la page les mots suivants «comme Adam qui donne [ ] ». Il convient de nous arrêter un instant sur cette incise gardant à l'esprit qu'elle se rapporte à la question de la langue comme nomenclature : Adam aurait donné les noms aux animaux et aux objets de la même façon que le Nomothète qui est cité dans le dialogue Cratyle de Socrate comme le premier qui a nommé toutes les choses. Il est évident que cette incise exprime à nouveau la critique saussurienne de la nomenclature car, pour Saussure, il serait inutile de penser au jour où la langue a surgi et où les objets ont été nommés :

L'acte par lequel, à un moment donné, les noms seraient distribués aux choses, par lequel un contrat serait passé entre les concepts et les images acoustiques - cet acte nous pouvons le concevoir, mais il n'a jamais été constaté. (SAUSSURE, 1967, p. 105).

On doit encore mettre l'accent sur le fait que, pour Saussure, le «troisième élément » est la conscience que l'individu qui parle a, qu'un mot spécifique s'applique à un être extérieur et non à l'objet en luimême. À ce propos, Chiesa (2008) explicite :

Dans l'association du signe (ou du sème) entre le signifiant et le signifié, Il y a un troisième élément, à savoir, non pas la chose elle-même, mais la conscience que le sème s'applique à un objet extérieur déterminé, de sorte que, en vertu de cette relation aux choses, le signe semble échapper aux lois et aux rapports de la langue.11 (CHIESA, p. 13-14, 2008).

Mais pour quel motif l'onymique échapperait aux lois générales du signe ? On peut supposer qu'à partir du moment où il y a un élément extérieur dans la constitution du signe linguistique, il ne pourra pas se lier négativement avec les autres signes du système. En outre, selon Chiesa (2008), le signe ainsi considéré avec la présence du troisième élément - cesse d'être de nature dyadique et passe à être triadique, coïncidant avec la conception d'Aristote. (cf CHIESA,p. 14,2008).

La continuation de cet extrait semble introduire, dans le phénomène de l'onymique, la catégorie des noms géographiques : 


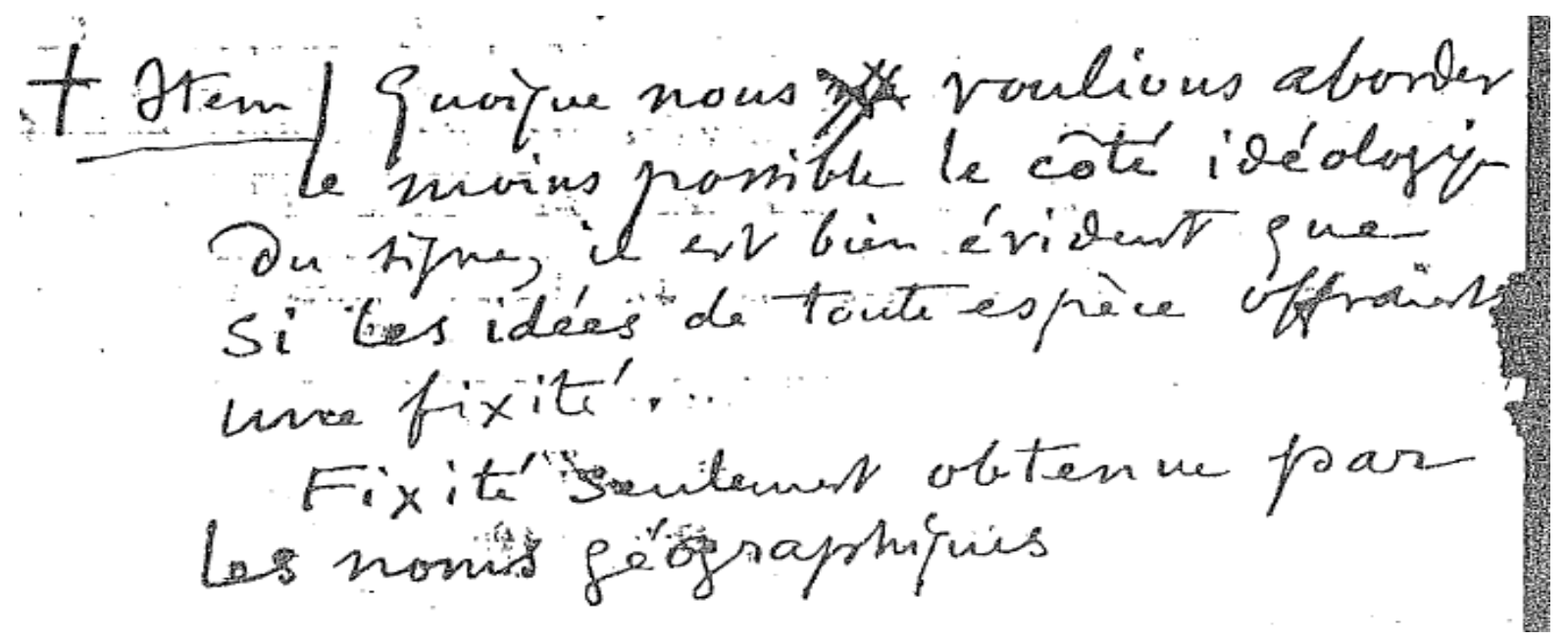

Figure 2. "Notes Item", p. 7

L'extrait ci-dessus ajoute deux nouveaux termes qui doivent être soigneusement analysés. Le premier est « fixité » et le deuxième « noms géographiques ». Ces deux termes établissent un rapport entre eux dans la mesure où les noms géographiques ou toponymes présentent une certaine fixité par rapport aux objets auxquels ils se réfèrent. Ainsi, le nom d'un pays, par exemple, ne change pas constamment car cela ne convient pas aux habitants de ce pays et des pays qui l'entourent. En outre, il faut noter que cette " fixité », selon Saussure, n'est obtenue que par les toponymes et non par tous les signes d'une langue.

Cet extrait constate, encore une fois, que ces catégories linguistiques qui possèdent un lien avec les objets du monde échappent à la loi générale des signes dans la mesure où ils ne seraient pas soumis - c'est le cas des noms géographiques - à la théorie de la valeur, par exemple. En contrepartie, si tous les signes offraient cette « fixité », la langue serait une nomenclature, voire une liste d'étiquettes qui nomment des objets et, par conséquent, la notion de « valeur » serait impossible à concevoir. La suite du texte, Saussure présente les noms propres accompagnés de la notion de noms géographiques:

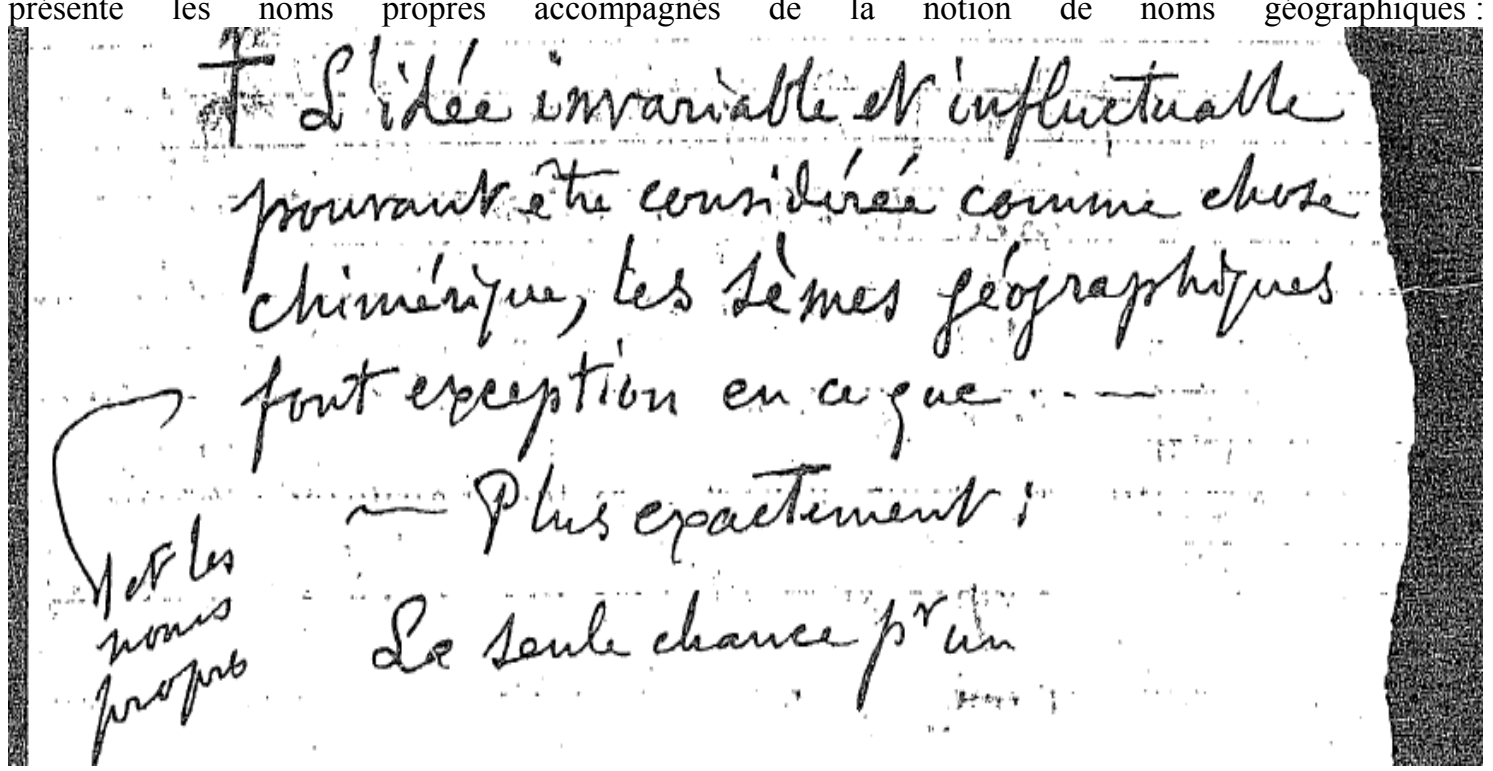

Figure 3. "Notes Item", p. 8

Comme nous pouvons le constater dans la Figure 3, les noms propres et géographiques, en offrant une propriété de fixité, font exception à ce qu'on pourrait déduire en ce qui concerne les lois générales des 
SHS Web of Conferences 1 (2012)
DOI $10.1051 /$ shsconf/2012010032

DOI 10.1051/shsconf/20120100327

Congrès Mondial de Linguistique Française - CMLF 2012
SHS Web of Conferences

signes, de plus, ils se présentent comme invariables et «influctuables» pour autant qu' ils ne se soumettent pas aux rapports internes du système. Finalement, le dernier extrait

L'idee os tt cela ent ta pivestion de Sarvin ti de míme quíun aposimi persiste hior on simes de mine un apos me intellecthel mouraiv pou fiant etts coustate'. Centain fue tans "Riono" ¿̇y a praindin 2 apromes courant parallilews Maing an fond pien ob Seivith fiosith puigun tion avair change' le nom des Rhone,

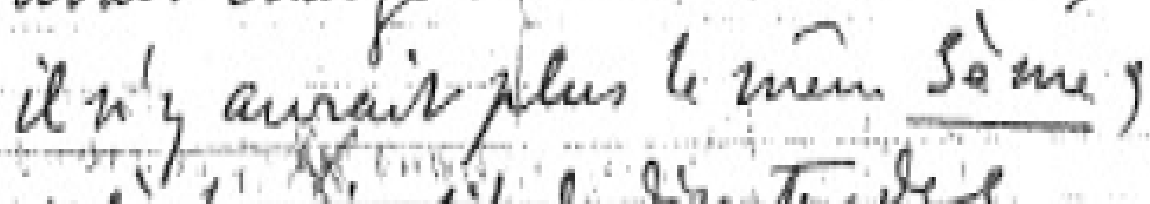

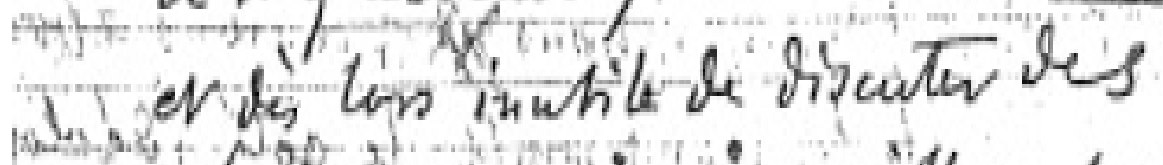

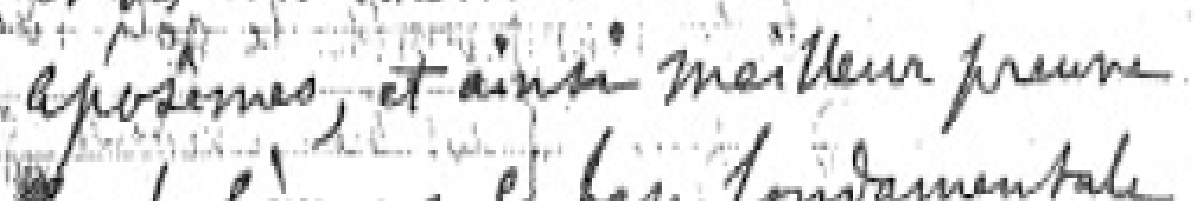
Yu le fem a fase fonimanantale ones le sima mativil cheiri'.

Figure 4. "Notes Item", p. 8

Article en accès libre placé sous licence Creative Commons Attribution 2.0 (http://creativecommons.org/licenses/by/2.0)

763 
L'exemple utilisé par Saussure dans l'extrait ci-dessus, semble mieux éclaircir le motif pour lequel les noms géographiques et les noms propres possèdent la propriété de fixité. Ainsi, le Rhône est un fleuve qui prend sa source dans les Alpes Suisses et se dirige vers l'ouest, jusqu'à la frontière entre la Suisse et la France. Ce fleuve possède le même nom depuis des siècles et donc, les habitants ne le connaissent que sous cette dénomination. Imaginons, cependant, qu'une autorité Suisse souhaite que le Rhône passe à s'appeler Rhésus. Les habitants des régions dans lesquelles le fleuve coule, n'accepteraient pas immédiatement le changement du nom et, s'ils l'acceptaient, continueraient à l'appeler Rhône, en mettant ce sème à côté de Rhésus.

Il est donc évident, que la catégorie linguistique du nom est d'une nature distincte de celle du signe dans le CLG. Partant du fait que les noms propres et géographiques constituent une exception aux lois générales du signe, ils ne devraient pas être partie prenante du contenu donné dans les cours de linguistique générale de 1907 à 1911, ce qui explique le silence sur ce thème dans l'édition de 1916.

\section{$4 \quad$ Les Niebelungen et les noms propres.}

Selon Starobinski (1974), les études saussuriennes sur les Niebelungen sont réunies dans environ dix-huit carnets - sans compter un certain nombre de feuilles -, l'ensemble catalogué sous les numéros Ms Fr. 3958 et Ms Fr. 3959 . Il est estimé que ces recherches ont été développées entre les années de 1903 et 1910 (cf. SATAROBINSKI, p. 9, 1974), comprenant ainsi les cours de linguistique générale donnés par Saussure de 1907 à 1911, à l'université de Genève.

Notre intérêt dans les recherches de Saussure sur les légendes germaniques se justifie dans la mesure où, en accord avec Starobinski (1974), il avait pour objectif de trouver la preuve que les personnages et les évènements légendaires possédaient une origine commune avec les personnages historiques. La question qui traverse cette enquête saussurienne se réfère donc à l'interférence des aspects extra-linguistiques dans les légendes. D'autre part, il convient d'affirmer que lorsque l'on se réfère à la relation qui existe entre les personnages légendaires et les personnages historiques, Saussure doit passer obligatoirement par la question du nom propre.

Dans ses travaux sur les anagrammes et sur la légende, le point de vue de Saussure s'elargit au champ sémiologique tout entier et assure le fondement d'une nouvelle science dite sémiologique, sans lequel toute l'explication du signe demeurerait un simple travail de linguiste historico-comparatiste du 19éme siècle. (SUNGDO KIM, p. 10, 1993).

Ainsi, les considérations sur les anagrammes et sur la légende rendent possible la constitution d'une réflexion sémiologique. On ne peut pas, de cet façon, isoler les trois courants de la pensée saussurienne le CLG, les anagrammes et les Niebelungen - sans que s'intallent relations théoriques :

La mythologie saussurienne n'était ni une simple curiosité égarée, ni une folie schizophrénique. Au fond, ce n'est pas le Saussure des anagrammes, car, d'une part, la mythologie saussurienne est épistémoloquement parlant proche du CLG, d'autre part, la mythologie était un domaine tout à fait habituel de l'indo-européaniste du 19siècle comme c'est le cas de Saussure. (SUNGDO KIM, p. 17, 1993). 
Saussure justifie as motivation pour l'étude des légendes germaniches quand il affirme que c'est nécéssaire connaître les processus naturels pour lesquels l'épopée est établie, c'est-adire, il y a une motivation théorique dans ses considérations :

Notre temps et notre société ayant perdu le sens de l'épopée, on voit tâtonner à son égard comme autour d'un objet inconnu, ce qui est tout naturel, mais précisément cela interdit de prononcer des excommunications contre aucune façon, je ne dis pas de la concevoir, mais de l'étudier, afin de se faire une idée des procédés naturels qui ont pu présider à l'épopée. (F. De Saussure Ms. Fr. 3958-7, 35v. apud SUNGDO KIM, p. 0, 1993)

Sur ce point, il est plausible d'affirmer que la question qui traverse les recherches sausuriennes sur les Niebelungem se rapporte à l'interférence d'aspects extra-linguistiques dans les légendes. De plus, en analysant la relation existante entre les personnages de légende et les personnages historiques, Saussure doit obligatoirement passer par la question du nom propre. De cette façon, le linguiste explique dans ce qui serapporte au nom propre que

Surtout voir que le nom n'est qu'um des éléments a.b.c.d placés exactement sur le même rang; car c'est là sans doute ce qui voile le plus la vérité de ce que nous essayons d'affirmer; on ne fera jamais admettre à certaines personnes que dans "Dietrich" le nom est indifférent ; qu'il n'a d'importance que s'il ne s'y ajoute une somme determinée de traits qui, venant à manquer, nous laisseraient devant le vide absolu (...) Ce sont les mêmes qui ne croiraient pas nécessaire de dire qu'ils font une même chose de Grudun l'épouse navrée de Sigurd et Kriemhild s'appuyant ici juste à l'inverse sans en rendre expressément compte a leurs lecteurs ou à eux-mêmes sur les caractères independants du nom. Aussitôt qu'on voudrait réduire cette conduite contradictoire à une méthode, il faudrait bien en venir à voir, de proche en proche, quelle est la nature fondamentale des êtres sur lesquelles [sic] raisonne la mytographie. À reconnaître que le nom a juste autant ou juste aussi peu de valeur. (SAUSSURE, apud AVALLE, p. 34, 1973).

L 'on s'aperçoit que Saussure effectue une approche entre la catégorie des noms et les signes linguistiques présents au CLG. De ce point de vue les noms seraient soumis aux mêmes relations que nimporte quels autres signes de la langue, ce qui est bien différent de l'affirmation selon laquelle les noms propres et géographiques "échappent aux Lois générales du signe" - comme cela a été dit dans le "Notes Item". Ainsi, les noms propres possèderaient une certaine valeur dans la mesure où ils sont en relation avec les autres signes du système, cependant, il semblerait, les noms propres ne seraient pas complètement dénués de positivité, considérant la fixité qu'ils présentent.

Dans d'autres extraits de ses remarques, Saussure affirme que les noms seraient des symboles. Si nous nous reportons au concept...de symbole au CLG, nous nous apercevons que la notion de symbole est distincte de la notion de signe: "Le symbole a pour caractere de n'être jamais toute à fait arbitraire; Il n'est pas vide, Il y a um rudiment de lien entre le signifiant et le signifié. Le symbole de la justice, la balance, ne pourrait pás être remplacé par n'importe quoi, un char, par exemple" (SAUSSURE, P.101, 1967). Ainsi, le signe possède la caractéristique d'être arbitraire et sa valeur est établie en fonction des relations établies avec les autres signes, alors que le symbole, selon le CLG, possède une certaine motivation qui l'empêche de se mettre en relation avec les autre signes de forme négative et différencielle. 
Pourtant, comme nous l'avons vu, Saussure met en rapport les notions de symbole et de signe dans le cas des légendes germaniques: “ces symboles, sans qu'il s'en doutent, sont soumis aux mêmes vicissitudes et aux mêmes lois que toutes les autres séries de symboles, par exemple les symboles qui sont les mots de la langue" (SAUSSURE apud AVALLE, p. 31, 1973). Il est donc possible d'affirmer que les différentes conceptions du mot symbole et les analyses différentes que Saussure lui a destiné, reflètent des moments différents au long de ses études. $\mathrm{Si}$, dans un premier temps, les noms propres et géographiques étaient distincts des signes linguistiques du fait qu'ils possédaient une fixité, plus tard, ils sont considérés comme symboles et sont toujours soumis aux mêmes vicissitudes que les autres. $\mathrm{Vu}$ que le CLG ne fait aucune remarque à propos de cette catégorie linguistique, il y a cependant dans un troisième temps Les symboles, à leurs tours, acquérissent le statut de "motivés", ou bien, ne seraient pas retenus dans la catégorie de signe linguistique. En d'autres passages, Saussure affirme qui :

Le nom n'a ni plus ni moins d'importance que tout autre côté. Il n'est pas comme chez un individu vivant une étiquette sur la personne, mais au même rang que les autres choses, et à ce point de vue plus important ; seulement ce qui compense, c'est que tandis que les autres caractères de l'individu sont inséparables de lui, et restent la base ferme de son identité même s'il change de nom, tout trait de l'être légendaire peut se dissiper au premier souffle avec autant de facilité que le nom. (SAUSSURE, apud PROSDOCIMI, p. 64, 1983).

Une fois de plus, Saussure rapproche la notion de nom propre de celle de signe linguistique et nie même la conception. Selon laquelle la langue est une nomenclature, ou encore, une liste de noms qu'i se réfèrent à une liste d'objets. Il semblerait que Saussure nie que le nom occupe une place spéciale parmi les autre signes de la légende. Selon Ferrandiz (2000), la tradition mytho-graphique de cette époque-là, semblait ressentir une prédisposition en faveur du nom propre légendaire...en ce qui concerne l'hypothèse qu'il aurait la capacités de récupérer complètement les caractéristiques des personnages. Cependant Sausurre ne semble pas être d'accord avec ce point de vue, car pour lui, les attributs seraient incorporés aux propres personnages et non pas à leurs noms.

Malgré le fait que le nom propre légendaire se rapporte aux personnages des légendes - Au cas où la relation de référence serait acceptée -, Saussure NE semble pas le concevoir comme inférieur au nom propre des langues naturelles. Cependant nous devons garderà l'esprît, que nous sommes face à deux catégories linguistiques différentes. À ce sujet Ferrándiz (2000) affirme que :

si en la lengua un nombre propio arbitrarieamente etiqueta una colección de rasgos de su referente humano, en la leyenda dichos rasgos no son menos arbitrarios que el nombre, y en todo momento pueden desprenderse de él - y él de ellos - y llevar una vida autónoma en el tejerse y desterjerse de las versiones legendarias. (FERRÁNDIZ, p. 188, 2000).

Quand il analyse la transmission d'une légende d'une communauté linguistique à l'autre alors qu'elle est fait partie d'une époque, Saussure affirme que la mémoire, ou plutôt, le manque de mémoire, exerce un rôle important dans les changements diacroniques auxquels les légendes dépendent. Il est interessant de noter que la catégorie linguistique des noms reçoit une influence directe de ce qui touché le facteur "mémoire" :

Catégorie de formation ingéniuse provoquées par le (défaut) de mémoire. Il s'agit des domaines lexicographiques comme ceux des noms de plantes, (noms minéraux, nom de petites bêtes, n'étant qu'à moitié appris de la masse des sujets parlants): connus seulment ()

Et alors, sans que le nom cesse d'être transmis, il est soumis à une loi de transformation totalement différent de celui du mot ordinaire... et qui aboutit à des 
séries d'étymologies compactes. (Ms. Fr. 3959-3, 2v; cité par PROSDOCIMI, 1983, P. 57-58)

Sur ce point, Saussure cite des noms de plantes, minéraux et animaux. Ces noms sont les noms des espèces qui possèdent une nature distincte des noms propres. Alors qu'un nom propre désigne probablement un objet déterminé dans le monde, les noms de l'espèce quand à eux désignent des classes d'objets. Il convient donc de faire quelques remarques au sujet de la nature de ces catégories linguistiques. Observez les deux phrases ci-dessous :

(a) Ângela est une enfant heureuse.

(b) La table est en bois.

Dans ces deux phrases les noms "Angela" e "table" désignent des objets. Nous pouvons affirmer que ceci est la caractéristique fondamentale de la catégorie linguistique des noms...: être pour l'objet, ce qu'il veut et quel qu'il soit (BRITO, 2003, p. 25). Pourtant il y a différentes façons pour un nom de désigner un objet. Dans la phrase (a) par example, en prononçant le nom Ângela, je me réfère à une personne déterminée qui a été nommée ainsi sans motiffforme aléatoire. D'autre part en prononçant la phrase (b), le mot "table" se réfère à toute sorte d'objets qui possède les caractéristiques inhérentes à une certaine classe. Ainsi, dans la seconde phrase la référence du nom peut représenter n'importe quel objet qui possède les caractéristiques d'une table et non pas une table en particulier.

Ainsi, les noms propres seraient noms qui, comme nous l'avons vu, ont une, dénomination imotivée et désignént un objet determinée. Il n'y a pas, donc, aucune caractéristique de "angelice" qui motive le nom de l'enfant. Les noms de espèce, au contraire, désignent tous les objets qui ont caractéristiques déterminées pour être classifiée chez une espèce. (cf. BRITO, 2003, p. 27). De cet façon, un objet seulement sera denominée comme "table" si il possède les caractéristiques de cet espèce.

$\mathrm{D}$ 'autres termes seront également abordés comme appartenant à la catégorie des noms si nous acceptons que dans ce cas il s'agit de se référer aux objets. Ainsi les indicateurs de subjectivité dans la terminologie Benvenistienne - comme "je" et "tu" et des expressions deitiques comme "ceci" ou "cela" désignent aussi un objet lorsqu'ils sont proférés dans une phrase. Les termes de masse comme (eau, or) et les descriptions définies aussi font également partie de la catégorie des noms et sont cités par Saussure comme soumis à un manque de mémoire. Les termes de masse se caractérisent par le fait qu'ils sont distribués dans des lieux divers à travers le monde en même temps: l'objet "eau", par exemple peut être rencontré dans régions différentes mais c'est cependant le même objet, il a la même composition chimique etc. Quant aux descriptions définies, elles possèdent des attributs qui définissent l'objet auxquel elles se réfèrent. Ainsi, la description définie "la capitale de la France" se réfère à Paris.

Ainsi, il nous semble que les noms propres liés aux legendes se classeraient mieux dans le contexte sémiologique plutôt que dans celui des noms propres appartenant aux langues naturelles. De plus, nous percevons un certain rejet de la part de Saussure en ce qui les concerne, cependant, même s'il l'évite, le problème de la relation entre les noms et les objets apparaît dans ses études.

En ce qui concerne le rôle du nom propre dans les observations saussuriennes liées aux légendes germaniques, nous pouvons ajouter, que celui-ci tient une place spéciale dans le domaine de la sémiologie.

\section{Conclusion}

À partir des aspects analysés dans le manuscrit saussurien « Notes Item », nous pouvons donc conclure que, le nom propre n'a pas été exclu de la théorisation saussurienne, au contraire, il y a des remarques 
importantes de Saussure dans les manuscrits qui expliquent la question du rapport entre les noms et les objets du monde.

En second lieu, nous concluons que critiquer la conception de langue comme nomenclature n'implique pas le fait de nier que certaines catégories linguistiques exercent la fonction de nommer certains objets dans le monde. Ceci est corroboré par les considérations saussuriennes à propos du phénomène de l'onymique et des noms propres. Em plus, nous pouvons affirmer que le role du nom propre dans lês considérations saussuriennes sur les lègendes, c'est un rôle très important dans le champ de la sémiologie.

Un autre aspect à souligner est le fait que si nous considérons ces trois tendances de la pensée saussurienne - o Notes item, le CLG et les Niebellungen - comme appartenant au domaine de la sémiologie, il est possible de tracer d'importants points d'intersection et de distance théorique dans les remarques saussuriennes car, si dans Notes item la catégorie linguistique des noms propres occupe une place notoire et possède assurément la qualité de fixité en relation aux objets auxquels elle se rapporte, au CLG, par contre, les noms propres n'occupent pas plus d'espace qu'un paragraphe dans la théorie saussurienne; il existe là, un certain détachement de cette catégorie linguistique par rapport aux autres signes saussuriens.

Il nous apparaît comme évident ici que l'étude des légendes germaniques exerce un rôle fondamental lorsque l'on considère le nom propre du point de vue de Saussure. Dans une certaine mesure, le nom propre est caractérisé comme le sont les signes linguistiques au CLG. Sa valeur sera également établie en fonction des relations avec les autres signes du systhème (a-b-c-d). Cependant, Il est posé comme um symbole et, si nous nous repportons aux observations de Saussure du CLG, nous nous apercevrons que la valeur d'un symbole n'est pas determinée par les autres signes de la langue dans la mesure où elle est motivée et non pas arbitraire.

Or, l'étude des légendes germaniques a eu lieu simultanément avec les cours de linguistique générale dispenses par Saussure à l'Université de Genève. Ceci nous porte à croire qu'il existe la possibilite que Saussure ait caché ses recherches sur le Niebelungen à ses eleves -ou peut-être en a-t-il parlé de forme superficielle- compte tenu qu'elles l'aurait amené à parler d'aspects sémiologiques beaucoup plus complexes et qui auraient certainement mis à jour/conduits à la question de la relation entre les noms et les objets.

Enfin, il est possible d'affirmer que, si au début de ses considérations Saussure adopte comme exemple des noms communs, tels quels pierre, vache, ciel, etc., ces exemples semblent être abandonnés au cours de son argumentation car ils sont remplacés par les noms géographiques et les noms propres. Cela explique, peut-être, le sentiment d'inconfort ressenti quand on comprend que des exemples de même nature que ceux utilisés dans le CLG - qui mettaient en évidence l'ordre propre à la langue et l'absence d'interférence d'un ordre extérieur - ont été utilisés au sujet du phénomène de l'onymique.

Ainsi, nous estimons que l'étude de la question des noms propres et géographiques et en conséquence, du rapport de référence, convient parfaitement aux études du langage, notamment si l'on considère qu'il existe encore plusieurs manuscrits qui peuvent élucider le problème du rapport entre les signes et les objets, du point de vue saussurien.

\section{Références bibliographiques}

AVALLE, D’A. S. La sémiologie de la narrativité chez Saussure. In : BOUAZIS, C., AVALLE, D'A.S. Essais de la théorie du texte. Paris, Galillée, p. 17-49, 1973 
BOUQUET, S. La sémiologie linguistique de Saussure : une théorie paradoxale de la référence?. Langages, $\mathrm{n}^{\circ} 107$, Paris, Larousse, 1992.

BRANQUINHO, J., MURCHO, D. GOMES, N. Enciclopédia de termos lógicos-filosóficos. São Paulo: Martins Fontes, 2006.

BRITO, A. N. Nomes próprios: semântica e ontologia. Brasília: Editora UNB, 2003.

CARDOSO, S.H.B. A questão da referência: das teorias clássicas à dispersão de discursos. Campinas, Autores Associados, 2003.

CHIESA, C. Saussure, Aristotel et l'onymique. In : Cahiers Ferdinand de Saussure. Revue suisse de linguistique générale, n.61. p.5-21. Publicado por Cercle Ferdinand de Saussure. Genève: Librairie Droz S.A., 2008.

FERRÁNDIZ, R. R. La semiología del nombre propio en Saussure : los Nibelungen y los Anagrammes. Revista de Investigación Linguística, n. 1, vol. 3, p. 181-205, 2000.

FLORES, V. BARBISAN, L. B. Sobre Saussure, Benveniste e outras histórias da linguística. In: NORMAND, C. Convite à linguística. São Paulo: Contexto, 2009.

FREGE, G. Sobre o sentido e a referência. In: Lógica e filosofia da linguagem. São Paulo: Cultrix, 1978.

GANDON, F. L'ambivalence théorique dans la recherche saussurienne sur la l'genede et les Notes item. Semiotica 115, 1-2, p. 173-190, 1997.

GADET, F. Saussure: une science de la langue. Paris: Presses Universitaire de France, 1990.

GARY-PRIEUR, M-N. Le nom propre constitue-t-il une catégorie linguistique?. Langue Française, Vol 92, p. 4-25, 1991.

GODEL, R. Les sources manuscrites du Cours de Linguistique Générale de F. De Saussure. Ge nève, Librairie Droz, 1969.

GUIMARÃES, E. Os limites do sentido: um estudo histórico enunciativo da linguagem. Campinas: Pontes Editores, 1995.

HENRIQUES, S. M. A categoria dos nomes e a referência: um estudo linguístico-filosófico. In: Horizonte Científico. Uberlândia, 2011.

KIM, S. La mythologie saussurienne: une nouvelle vision sémiologique? (a propos de la continuité de la pensée saussurienne). Semiotica 97, 1-2, 1993.

KLEIBER, G. Sens, référence et existence : que faire de l'extralinguistique?. Langages 127, 1997.

PAFUME, M. C. O último curso de Ferdinand de Saussure e a sua presença no "curso linguística geral". In: Revista Entrepalavras. Fortaleza, vol. 1, n. 1, p. 59-69, ago/dez-2011.

SAUSSURE, F. de. Curso de Linguística Geral. [1916] Editado por Charles Bally \& Albert Sechehaye com a colaboração de Albert Riedlinger. Tradução A. Chelini, J. P.Paes e I. Blikstein. 5a. ed. São Paulo: Cultrix, 1973.

. Notes Item. Sôme et sème . In 'Papiers Ferdinand de Saussure', 3951: 'Notes de Linguistique Générale'. Bibliotèque de Genève, 1891. 
Cours de linguistique générale: édition critique par Rudolf Engler. Tomo 1, Otto Harrassowitz Wiesbaden; 1968-1989.

Cours de linguistique générale: édition critique par Tullio de Mauro. Paris, Payot, 1986.

SILVA, K. A. Saussure e a questão da referência na linguagem. 141 p. Dissertação de Mestrado - UNICAMP. Campinas: 2008. Disponível em: <http://www.bibliotecadigital.unicamp.br/document/?code=000435701 >

SILVEIRA, E. As marcas do movimento de Saussure na fundação da linguística. Campinas: Mercado das Letras/FAPESP, 2007. 168 p.

SOFIA, E. Sur le concept de "valeur pure". In: Revista Letras \& Letras, vol. 25-1. Uberlândia: Edufu, 2009.

STAROBINSKI, J. As palavras sob as palavras. São Paulo, Perspectiva, 1974.

TESTENOIRE, P.Y. Le nom propre em débat au tournant du siècle (Whitney -Bréal - Saussure). Histoire, épistemologie, $\quad$ réflexivité. $\quad$ Paris, $2008 . \quad$ Disponível $<\mathrm{http}: / /$ www.linguistiquefrancaise.org/articles/cmlf/pdf/2008/01/cmlf08040.pdf $>$

VINHAIS, E. A. Saussure: uma teoria e dois destinos? In: Revista Eutonomia. ano 3,vol. 2. dez 2010.

WILMET, M. Arbitraire du signe et nom propre. Cahiers de linguistique hispanique médiévale. N. 7, vol. 7, p. 833842,1988

${ }^{1}$ Désormais nommé CLG.

${ }^{2}$ Le nom propre apparaît seulement dans le CLG dans le chapitre où Saussure se consacre à l'analogie. Cet extrait sera cité au long de cet article.

${ }^{3}$ Nous avons pris connaissance des résumés de ces deux communiqués à travers l'œuvre « Recueils des publications scientifiques de Ferdinand de Saussure », éditée par Léopold Gautier et Charles Bally,qui contient la production scientifique du linguiste genevois durant sa vie.

${ }^{4}$ Les manuscrits utilisés dans ce travail ont été sélectionnés et reproduits par la Professeur Docteur Eliane Mara Silveira, durant son séjour à Genève, entre le 13 et le 24 juin 1999, grâce à l'aide financière du « Fundo de Apoio a Ensino e à Pesquisa (FAEP-UNICAMP).

${ }^{5}$ Les manuscrits sont archivées dans la Bibliothèque de Genève sur le numéro de code Ms. Fr. 3958. Notes et brouillons sur les Nibelungen 8 cahiers dans un carton de 260x205 mm Total des feuillets écrits: 386f. (Total par cahiers) e Ms. Fr. 3959 Notes et brouillons sur les Nibelungen (suite). Tristan. 9 cahiers +4 enveloppes dans un carton de $350 \times 275 \mathrm{~mm}$. Total des feuillets écrits: $431 \mathrm{ff}$ (fol. Par cahiers). (SUNGDO KIM, p. 12, 1993). Malhereusement, nous n'avons pas contact directe avec ces documents.

${ }^{6}$ Nous considérons que l'arbitraire est le príncipe qui oriente le desligamento de la référence et non pas la théorie de la valeur, vu que si le rapport entre le signifiant et le signifié n'était pas arbitraire, "la valeur perdrait em partie son caractere, car elle contiendrait um élément imposé de l'extérieur”. SAUSSURE, p 132, 1973).

${ }^{7}$ Nous avons utilisé le terme "débrancher" pour remplacer celui “d'exclusion” vu que nous ne considérons pas que le phénomène de référence ait été exclu des remarques de Saussure.

${ }^{8}$ En accord avec Silva (2008), cette distinction établie par Gadet (1990) proportionne la perception du déplacement du principe de l'arbitraire effectué par Saussure. 
(C) aux auteurs, publié par EDP Sciences, 2012 